\title{
Contribution du SIG à la Prévention et à la Gestion des Risques d'inondation dans le District de Bamako au Mali
}

\author{
Oumar Dembélé, PhD \\ Docteur en Géographie Urbaine, Chercheur au \\ Centre de Recherche sur le savoir local - Point Sud de Bamako, Mali \\ Issa Ouattara, PhD \\ Docteur en Géographie de l'Environnement, Enseignant-Chercheur à \\ l'Institut de Développement Economique et Social de Bamako, Mali
}

Doi:10.19044/esj.2019.v15n30p256 URL:http://dx.doi.org/10.19044/esj.2019.v15n30p256

Résumé

En raison de sa situation géographique, la ville de Bamako reste fortement vulnérable aux risques d'inondations. La présente étude a pour objectif de mettre en exergue un outil opérationnel et évolutif d'aide à la prise de décisions en matière de prévention et de gestion des risques d'inondations dans le District de Bamako en République du Mali. Il s'agit d'élaborer à partir du Système d'Information Géographique des grilles d'analyse et d'évaluation permettant aux décideurs de mieux appréhender les risques naturels. La méthodologie adoptée s'est appuyée sur une approche par télédétection appuyée par des enquêtes et relevés de terrain. Les résultats de l'étude ont montré que les nombreux aménagements réalisés à travers la ville grâce aux politiques publiques urbaines, ont entrainé d'importants changements spatiaux-temporels. Toutefois, des occupations anarchiques règnent toujours dans la ville de Bamako, notamment sur les berges des cours d'eau qui constituent à priori des zones inondables. Des constructions existent dans les zones à risque où la vulnérabilité des populations reste forte. Les cartes réalisées indiquent les zones à stress et orientent les décideurs dans les options d'aménagement pour une réduction des risques d'inondation dans les années à venir au travers de la planification urbaine et de la participation des populations touchées dans l'élaboration des stratégies d'adaptation.

Mots-clés: Bamako, Prévention, Gestion, Risques, Inondations, SIG 


\title{
GIS Contribution to the Prevention and Management of Flood Risks in the District of Bamako in Mali
}

\author{
Oumar Dembele, PhD
}

Doctor in Urban Geography, Researcher at the

Center for Research on Local Knowledge - Southern Point of Bamako, Mali

\author{
Issa Ouattara, PhD
}

Doctor in Environmental Geography, Teacher-Researcher at the Institute of

Economic and Social Development of Bamako, Mali

\begin{abstract}
Due to its geographical location, the city of Bamako remains highly vulnerable to flood risk. The objective of this study is to highlight an operational and evolving decision support tool for flood risk prevention and management in the Bamako District of the Republic of Mali. This is to develop from the Geographic Information System grids analysis and evaluation for decision-makers to better understand natural risks. The methodology adopted was based on an exploratory approach sustained by field surveys and the analysis of cartographic data. The results of the study showed that the numerous developments carried out throughout the city by urban public policies have led to a great spatial "metamorphosis". However, anarchic occupations are still a reality in the city of Bamako, especially on the banks of rivers are a priori vulnerable areas. Buildings exist in risk areas where people's vulnerability remains high. The maps produced indicate areas of stress and guide decision makers in management options for flood risk reduction in the coming years through urban planning and the participation of affected populations in the development of adaptation strategies.
\end{abstract}

Keywords: Bamako, Prevention, Management, Flood, GIS

\section{Introduction}

Les populations du District de Bamako en République du Mali sont fortement vulnérables face aux risques d'inondation. Les inondations occasionnent des conséquences et des coûts sur l'opinion publique qui entraînent une préoccupation grandissante au niveau des autorités locales. Pourtant, les stratégies mises en place par les autorités urbaines pour faire face aux risques d'inondation dans la ville de Bamako, semblent peu efficaces. 
Le contraste est souvent saisissant entre les moyens mis en œuvre pour la connaissance de l'aléa et l'indigence des travaux sur les vulnérabilités, définies par Hubert et Ledoux (1999, p.25) comme « la mesure des conséquences dommageables de l'inondation sur les enjeux » soit «la fragilité d'un système socio-économique dans son ensemble face au risque ». Ainsi, la loi du 30 juillet 2003 relative à la prévention des risques et à la réparation des dommages prône parmi ses idées fortes, la réduction de la vulnérabilité des biens et des personnes par la révision des conceptions urbanistiques, des modes d'urbanisation et de la cartographie réglementaire qui doit découler non de la seule étude des aléas mais, aussi de l'analyse de la vulnérabilité des territoires (Pottier, 2003, p.31). Cette politique de niveau national ne résout pas l'ensemble des problèmes auxquels les acteurs locaux sont confrontés.

Dans le cadre de la gestion locale du risque, les volontés tendent vers le développement d'outils et de méthodes permettant de mieux apprécier les différents aspects de la vulnérabilité dans l'approche du risque d'inondation (approche qui a longtemps privilégié les uniques études sur l'aléa). Néanmoins, l'expérience manque et il convient donc de développer des outils d'aide à la décision en matière de connaissance des vulnérabilités au niveau local pour la prévention des risques naturels.

La présente étude est une contribution à la géographie urbaine de Bamako. Elle vise à mettre en exergue le rôle des systèmes d'information géographique (SIG) dans l'analyse spatiale des paysages urbains et des sites naturels (cours d'eau) occupés. Le SIG reste un outil d'évaluation du risque et joue pleinement un rôle dans l'aide à la prise de décisions et la gestion des stratégies d'adaptation des populations vulnérables. Sur un autre plan, on note l'existence de nombreux outils et documents stratégiques et de planification urbaine relatifs au développement urbain de cette grande métropole malienne. Il s'agit notamment du Schéma Directeur d'Aménagement et d'Urbanisme, des plans d'urbanisme sectoriel. Toutefois, ces documents restent inappliqués. Ceci fut aussi une source de motivation pour aborder un tel sujet.

\section{Matériel et méthodes}

\subsection{Présentation de la zone d'étude}

Capitale de la République du Mali, Bamako est située dans la partie Sud-Ouest du pays. Par l'ordonnance $\mathrm{n}^{\circ} 78-32 / \mathrm{CMLN}$ du 18 août 1978, modifiée par la loi n82-29/AN-RM du 02 février 1982, la ville de Bamako a été érigée en un District de six Communes. Ce District couvre une superficie d'environ $267 \mathrm{~km}^{2}$ pour une population estimée à 2384780 habitants (Direction Régionale de la Planification, de la Statistique, de l'Informatique, de l'Aménagement du Territoire et de la Population du District de Bamako, 2015), soit une densité de 8932 habitants au $\mathrm{km}^{2}$. Les sites de l'étude comprennent les quartiers de Banconi, Djicoroni-Para et Missabougou, 
respectivement situés en Commune I, en Commune IV et en Commune VI du District de Bamako. Leurs populations sont fortement vulnérables face au risque d'inondation. La carte 1 ci-dessous présente les sites de l'étude.

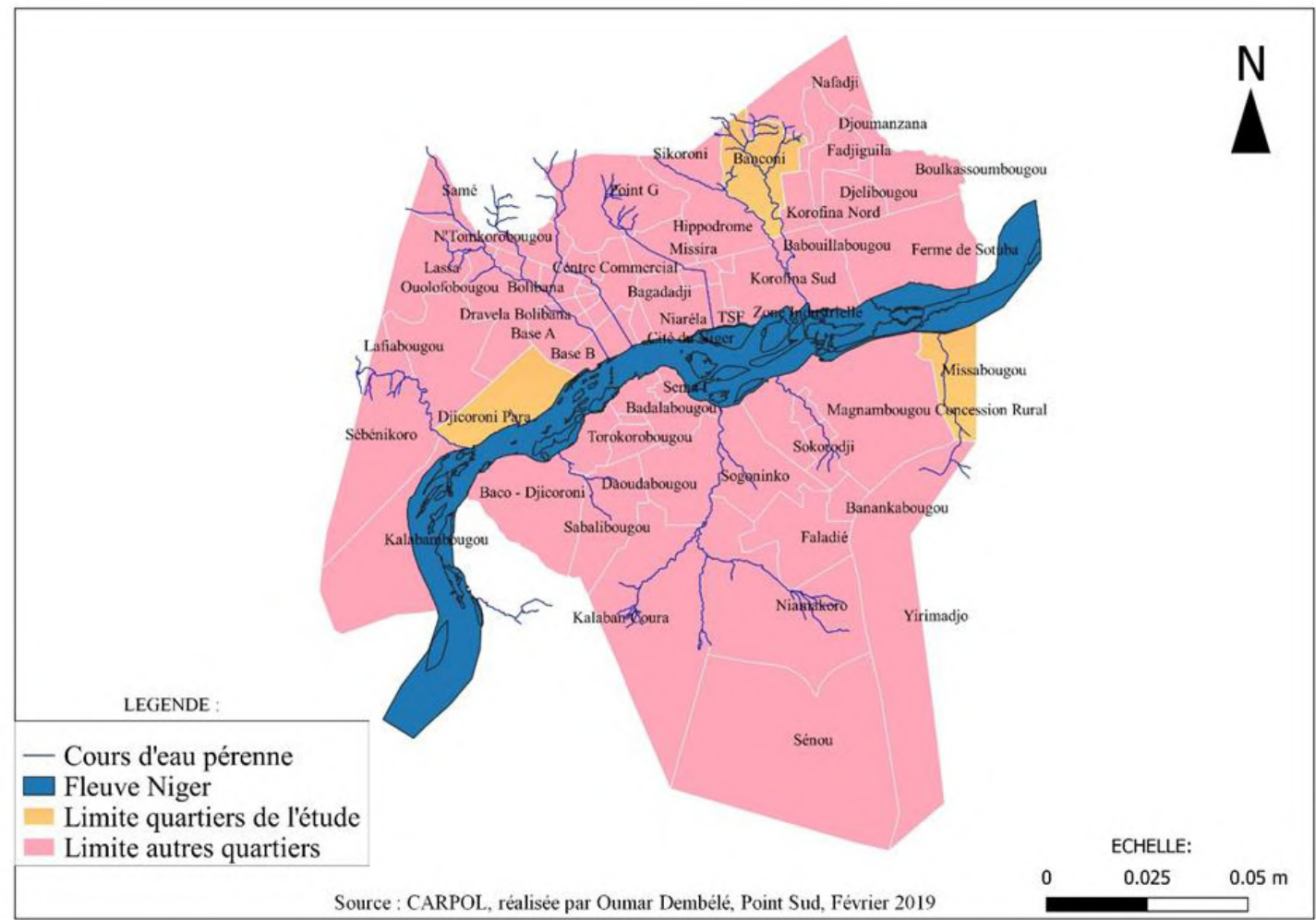

Carte 1: Localisation des sites de l'étude

La ville de Bamako est située dans le climat soudanien, qui par définition, est un climat chaud, toujours plus de $18^{\circ} \mathrm{c}$ (Mali-météo). La caractéristique principale du climat soudanien est l'alternance de deux saisons : une saison sèche longue, et une saison pluvieuse allant de Juin à Septembre. La saison sèche comprend une courte période fraîche (décembre à février) et une longue période chaude avec des extrêmes parfois trop élevées (plus de $38^{\circ} \mathrm{c}$ ). La température moyenne de Bamako est de $27,7^{\circ} \mathrm{C}$ avec une pluviométrie moyenne de $1100 \mathrm{~mm}$ (Mali-météo).

S'inscrivant presqu'entièrement dans un site naturel correspondant au grand bassin versant du fleuve Niger, l'agglomération de Bamako présente des sols argilo-limoneux ou sablonneux le long du fleuve et latéritique ailleurs. Par endroit, affleure la roche latéritique dure. La topographie de la ville est caractérisée par le découpage du site par le fleuve Niger d'Ouest en Est, par de nombreux marigots qui se déversent dans le fleuve et par des reliefs accentués. L'agglomération est constituée de deux parties nettement individualisées. Au nord, le site naturel de la rive gauche (7000 ha) très marqué 
s'inscrit d'une part entre le fleuve et les falaises des monts mandings (4 km en moyenne) et d'autre part, entre deux «verrous » où se rejoignent la falaise et le Niger : à l'Est au niveau de Moribabougou, à $15 \mathrm{~km}$ du centre-ville, à l'Ouest au niveau de Sébénicoro, à $12 \mathrm{~km}$ du centre-ville. Au sud, la rive droite occupe un site de 1200 ha depuis l'Aéroport international de Sénou (dont la piste s'envole et la zone de sécurité qui le prolonge, barrent le site d'Ouest en Est sur 16,5 km environ avec une largeur de 6,5 km environ) et les reliefs de Tienkoulou jusqu'au fleuve Niger.

$\mathrm{Du}$ point de vue géologique, le substrat reste le vieux socle précambrien, commun à toute l'Afrique de l'ouest. Ce socle, formé de roches métamorphiques en général, a été arasé par l'érosion qui y a déposé des sédiments. Le rebord du plateau sur lequel Koulouba et l'hôpital du «Point $\mathrm{G}$ » sont installés, est une strate de grès durs datant du primaire ou peut-être même avant, du supra-cambrien. Les grès du seuil de Sotuba « faiblement glauconieux sédimentaires dans la zone néritique » seraient d'origine marine. La plaine du haut Niger, dans laquelle la ville s'étend, est une couche de schistes tendres (Urvoy, 1942). Partout, le sol est latérite. Il s'agit des sols ferrugineux tropicaux, d'aspect rougeâtre, provenant des terrains sous-jacents (Urvoy, op cit.). La morphologie du site de Bamako, est liée à sa géologie. Cette partie de l'Afrique n'ayant subi que des mouvements tectoniques insignifiants, le relief est principalement constitué des couches géologiques que l'érosion différentielle a mises à nu. Celui de Bamako se résume en deux éléments : le plateau, partie nord du plateau mandingue n'est que la couche de grès supra-cambrien, que les mouvements hercyniens ont portée à l'affleurement. Il détermine un escarpement dominant le fleuve. L'allure générale est celui d'une bordure festonnée. Les festons constituent de véritables petites falaises au rebord abrupt. Il s'agit, en forme d'arc et d'Ouest en Est, de Lassakoulou (413 m), Koulounikokoulou (483 m), Koulouba (404 $\mathrm{m})$ et la corniche $\mathrm{du}$ «Point $\mathrm{G}$ » $(403 \mathrm{~m})$. Ces festons sont généralement séparés par de petits cours d'eau qui entaillent le plateau. C'est le cas du marigot «Woyowayanko » entre le Lassakoulou et le Koulounikokoulou ; du marigot «Sogonafing » entre le Koulounikokoulou et celui de Koulouba, etc. Les vallées de ces cours d'eau et le bas-fond au pied du plateau, constituent les réserves de végétation de Bamako. Ce sont donc des parties sensibles du site que l'extension des constructions menace sérieusement. Au pied du plateau, s'étend la plaine nigérienne, constituée de schistes. D'un point de vue hypsométrique (altitude), c'est un terrain presque régulier. On passe presque insensiblement, du pied de la falaise de Koulouba au fleuve : $340 \mathrm{~m}$ au niveau de l'ancien site de l'ENA (Ecole Nationale d'Administration) à seulement 320 $\mathrm{m}$ au niveau du fleuve et cela sur près de $2 \mathrm{~km}$. Au-delà du fleuve, la plaine présente quelques collines, guère importantes $(350 \mathrm{~m}$, ce qui fait $30 \mathrm{~m}$ de plus par rapport au fleuve), notamment à Badalabougou, Quartier-Mali et 
Sabalibougou. Ailleurs le terrain est plat. Il faut atteindre le sud-est du fleuve, vers Magnambougou, Sokorodji et Djaneguela, pour voir une dénivellation importante. A cet endroit le fleuve Niger rencontre pour la première fois le plateau dont les deux versants se rapprochent.

Avec une telle monotonie, on comprend facilement que les abords immédiats du fleuve soient des terrains inondables. Ils constituent donc une partie sensible du site à cause du risque auquel les populations sont exposées. Cependant, dans toute zone urbanisée, les qualités naturelles du site sont en train d'être dissimulées par le site récréé : occupation des plateaux et des versants par l'habitat. Ce qui favorise les inondations. Pourtant, de nombreuses personnes installées sur ces sites semblent minimiser le risque d'inondation auquel elles s'exposent. D'autres personnes, ayant obtenu des autorisations d'occupation des mairies, et même des titres fonciers pensent ainsi être à l'abri de tout risque. L'étude du cadre naturel des zones à risque d'inondation de Bamako n'a d'intérêt que si elle permet une compréhension des sites vulnérables face à l'occupation des sols (végétation et hydrographie), en rapport avec les activités de la localité.

\subsection{Démarche méthodologique}

La méthodologie adoptée pour l'étude s'est appuyée sur l'exploration documentaire, l'observation, la réalisation d'enquêtes de terrain et l'analyse des données cartographiques et des images satellitaires. L'exploration documentaire a consisté en la consultation des savoirs déjà produits sur la thématique. Des constructions anarchiques situées dans le lit et les servitudes des cours d'eau dans l'agglomération de Bamako ont été observées. Une typologie des maisons (en dures, semi-dures et en bancos) observée sur le terrain nous a permis d'évaluer la vulnérabilité des populations en cas d'inondation. Des maisons effondrées suite à des inondations et des caniveaux bouchés par les déchets (source d'inondation), ont également été visitées. Le District de Bamako compte soixante-huit quartiers répartis entre six Communes selon la Direction Régionale de la Planification, de la Statistique, de l'Informatique, de l'Aménagement du Territoire et de la Population du District de Bamako (DRPSIAP-DB, 2015). Compte-tenu du nombre important de quartiers, nous avons opté pour l'échantillonnage non probabiliste et retenu un échantillon de 300 ménages, soit 100 ménages par quartier. Banconi, Djicoroni-Para et Missabougou ont été retenus suivant le critère de fréquence des cas d'inondations ces dernières années dans ces quartiers. Les individus, principalement les chefs de ménage ou une personne adulte du ménage, ont été interrogées individuellement. Les catégories socioprofessionnelles suivantes ont été enquêtées : $37 \%$ de commerçants, $16 \%$ de ménagères, $13 \%$ de fonctionnaires de, $23 \%$ d'employés du secteur privé, $2 \%$ de pêcheurs et $9 \%$ d'artisans. Les données collectées portaient essentiellement sur les facteurs 
des risques d'inondation, les niveaux d'évaluation de la vulnérabilité des populations et leurs perceptions dans la gestion participative des risques. Grâce au logiciel SPSS, les réponses collectées ont été traduites sous forme de graphiques.

Le Système d'information géographique (SIG) a permis de procéder au traitement des données cartographiques à partir du logiciel Envi 4.7 et Arc GIS.9 en vue de constituer une véritable analyse spatiale. Nous avons consacré un temps pour les prises des coordonnées géographiques des habitations dans les communes ou quartiers concernés par l'étude et exposés au risque d'inondation à Bamako. Des données GPS ont été utilisées à travers le logiciel MapSource qui, traite les Waypoints ou journal du jour en fichier Excel contenant les coordonnées géographiques des bâtis occupant les berges. Ces coordonnées géographiques en fichier Excel appelées aussi les Points de contrôle sont destinées à faire le géo-référencement ou calage sur les fonds de cartes. Des entités ou couches thématiques relatives aux catégories de bâtis, des cours d'eau, de la localisation des bâtis dans le lit et les servitudes des cours d'eau ont été retenues pour l'évaluation de la vulnérabilité aux inondations. Cet outil a été d'un apport précieux dans l'évaluation de la vulnérabilité aux inondations selon plusieurs facteurs : (i) la vulnérabilité liée à l'aléa, (ii) la vulnérabilité de la population, (iii) la vulnérabilité du bâti et (iv) la vulnérabilité liée à la gestion de crise.

La disponibilité depuis 1972 des données Landsat, le plus ancien programme d'observation de la Terre, en fait une source documentaire exceptionnelle. En effet, les données de Landsat assurent une couverture globale de la Terre depuis 1972 grâce aux capteurs MSS (Multi Spectral Scanner, 1972-1992), TM (Thematic Mapper, depuis 1982 et toujours opérationnel en 2006 avec Landsat 5), et ETM+ (Enhanced Thematic Mapper, depuis 1999 et opérationnel en 2000 grâce à Landsat 7). Les données de Landsat permettent de suivre l'évolution globale de l'occupation des sols, notamment la progression des dunes sableuses, l'état de la végétation naturelle et la dynamique urbaine (Dia et al., 2006b). Les imageries satellitaires Landsat de 1986, 1972 et 2017 ont permis de comprendre la transformation progressive du paysage urbain de Bamako.

\section{Résultats et discussion}

\subsection{Résultats}

De sa fondation à la fin du XVIII ${ }^{\text {ème }}$ siècle, la ville de Bamako n'a cessé de changer de physionomie. 


\subsubsection{Dynamique urbaine diffuse et accentuation des risques d'inondation}

La présentation de l'agglomération de Bamako traduit une urbanisation diffuse (carte 2).

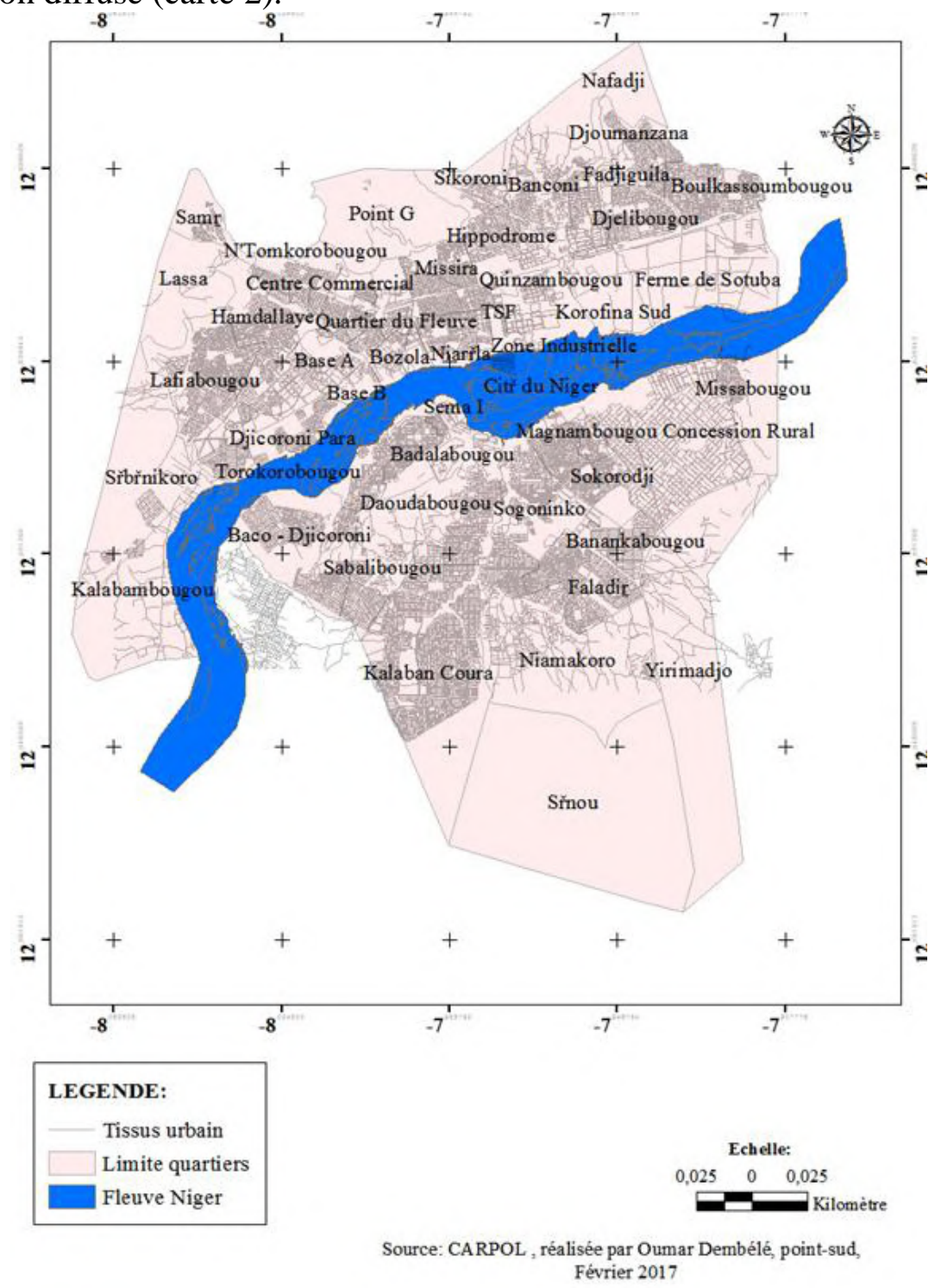

Carte 2 : Tissu urbain du District de Bamako

De sa fondation par les NIARÉ sur la rive gauche du fleuve Niger vers la fin du XVI ${ }^{\text {ème }}$ siècle à nos jours, la ville de Bamako a connu un essor démographique. De 4400 habitants à la fin du XVI ${ }^{\text {ème }}$ siècle, la ville compte aujourd'hui 1809106 habitants (RGPH, 2009). Cette dynamique démographique de Bamako a été accompagnée d'une dynamique spatiale. Les 
images satellitaires Landsat 1, 2 et 3 ci-dessous analysées permettent de faire un suivi de l'étalement urbain dans les zones dépressionnaires à risque élevé d'inondation entre 1972 et 2017.

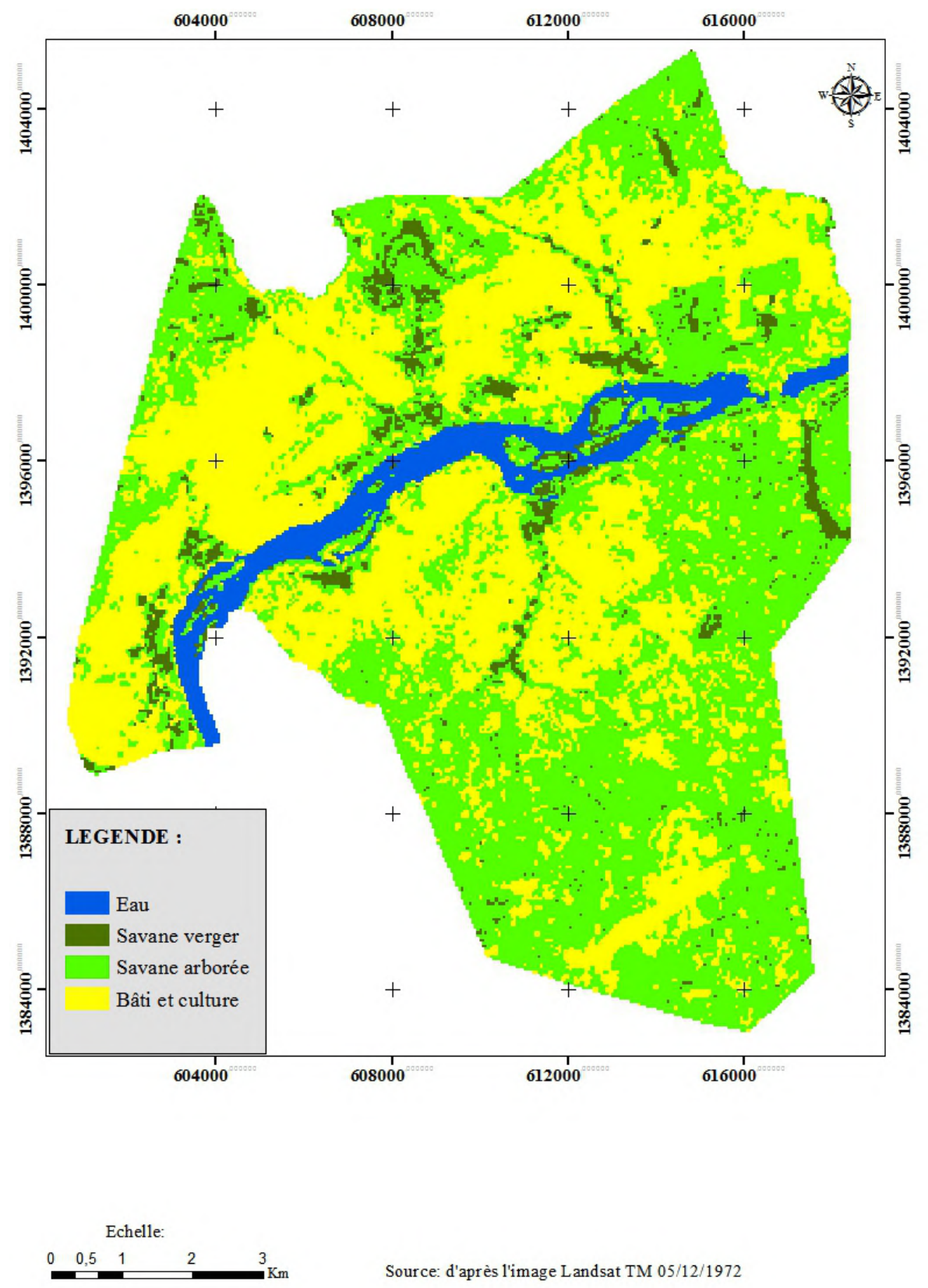

Image 1 : Occupation du sol urbain de Bamako, période 05/12/1972 


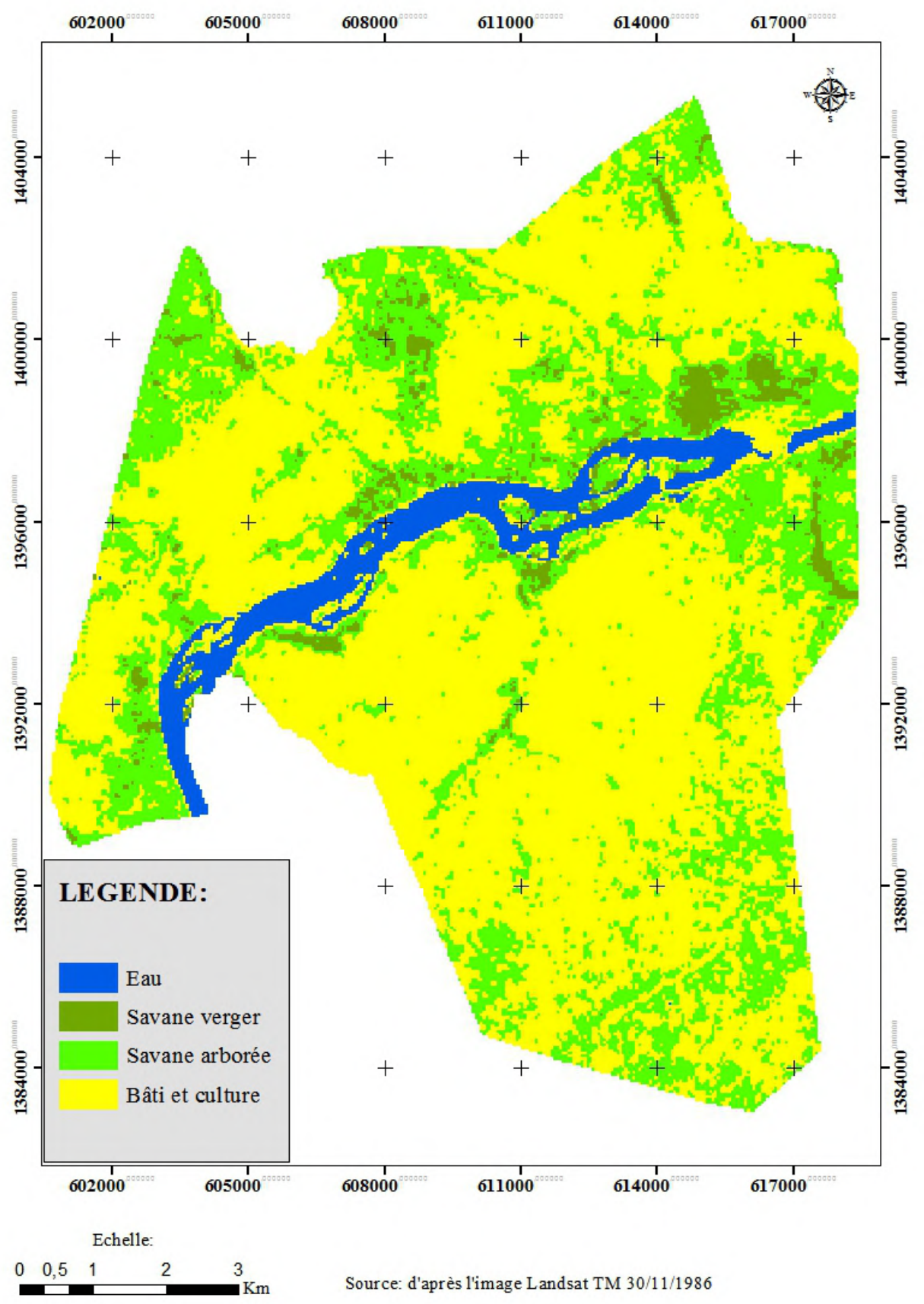

Image 2 : Occupation du sol urbain de Bamako, période 30/11/1986 


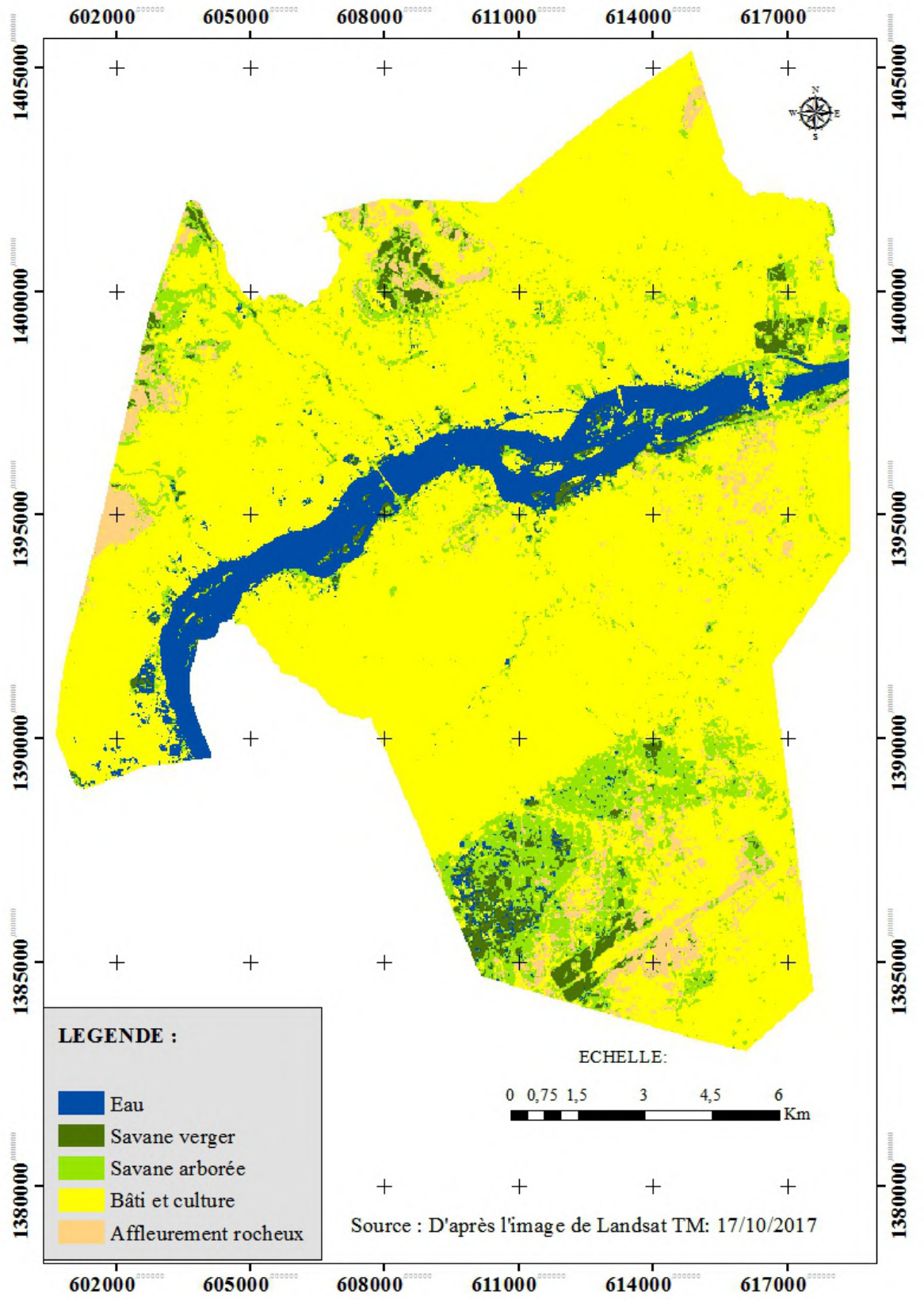

Image 3 : Occupation du sol urbain de Bamako, période du 17/10/2017

Les images ci-dessus confirment l'évolution de l'occupation des unités de terre du District de Bamako à travers les différentes dates prises. A la lecture de l'image de Bamako en 1972, elle révèle que très peu d'espaces étaient occupés dans le District de Bamako. Cependant, on peut observer une certaine diminution de la couleur verte (symbolisant la végétation). Ce qui 
signifierait qu'on a affaire à une extension spatiale des zones bâties à Bamako. Ceci est conforté par les résultats des recensements démographiques de la capitale malienne pendant la même période. "Le taux d'accroissement annuel observé durant la période 1968 - 1976 est impressionnant : $12 \%$. En effet, on passe de 170000 à 419239 habitants en seulement huit ans; un doublement de la population en moins de huit ans est un rythme de croissance trop rapide et assez rare » (Diarra, 1999, p.48). Quarante ans plus tard, la situation de l'occupation du sol nous offre une autre lecture. Les zones bâties se sont considérablement étendues. Par ailleurs, les obstacles topographiques (basfonds marécageux, plateaux et collines) ont guidé l'occupation du sol. Cependant, les éléments physiques vus comme contraintes sont à relativiser, en ce sens que, de plus en plus, les populations occupent illégalement et gratuitement ces endroits. A cela, s'ajoute la pauvreté et la politique de logement qui attire de plus en plus des populations vers les bas-fonds marécageux, plateaux et collines. Une telle prolifération des habitations se traduit par une occupation des espaces végétalisés.

\subsubsection{L'occupation anarchique des berges à Bamako}

De nombreuses personnes installées sur les sites d'inondation ne croient pas au danger auquel elles s'exposent. D'autres personnes, ayant obtenu des autorisations d'occupation des mairies, et même des titres fonciers se croient ainsi à l'abri de tous risques d'inondations. Dans cette logique d'exposition au risque, des années d'inondation ont été enregistrées. Ce sont successivement en 1992, 1999, 2002, 2012. Mais, de la mémoire collective des populations, celle survenue le mercredi 28 août 2013, est révélatrice du danger qui menace sans cesse des milliers d'habitants. Son intensité et l'ampleur des dégâts ont été impressionnantes. Plusieurs secteurs ont été littéralement submergés par le débordement des cours d'eau et les eaux collinaires venant des plateaux qui surplombent la ville. Les conséquences du sinistre sont désastreuses. On en dénombre 789 familles sinistrées regroupant 7077 âmes, 168 maisons détruites, des biens emportés et 3 décès (graphiques 1 et 2).

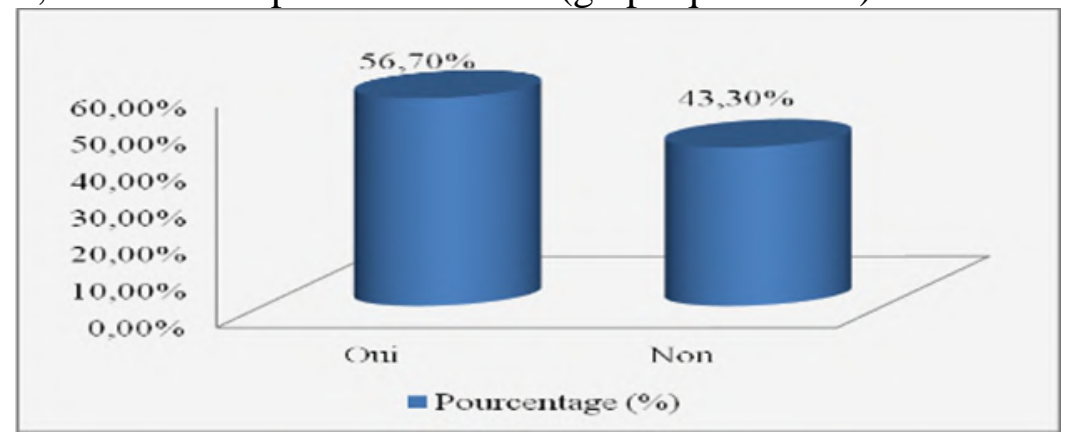

Graphique 1 : Ménages victimes des inondations en 2013

Source : Enquête de terrain, Juillet 2016 


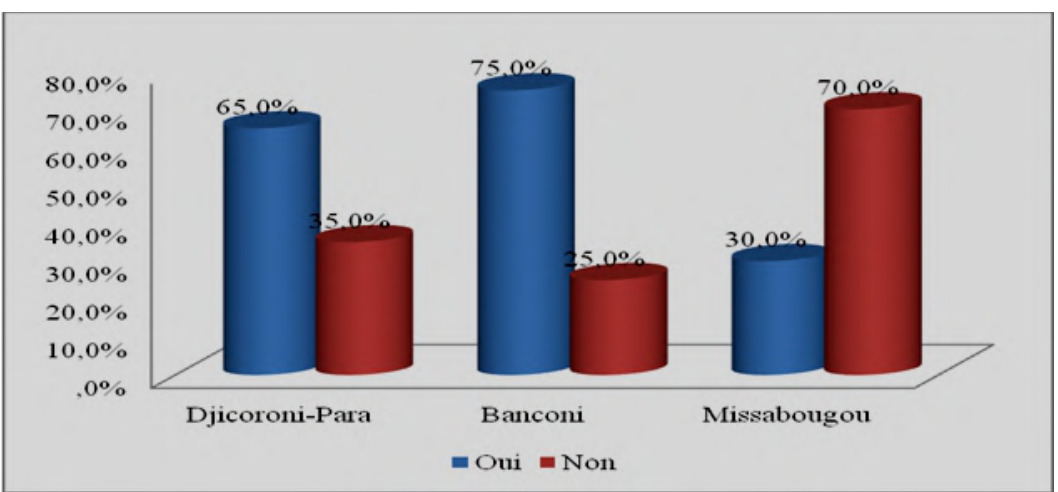

Graphique 2 : Ménages victimes des inondations de 2013 par quartier Source : Enquête de terrain, Juillet 2016

Les inondations ont provoqué à Bamako la mort de plus d'une trentaine de personnes, plus de 3000 sinistrés et laissé plus de 400 familles sans abris, selon les derniers bilans de la Direction Générale de la Protection Civile en 2013. En se référant aux graphiques (1 et 2) ci-dessus, 56,7\% de ménages enquêtés ont été victimes des inondations (cf. photos 1 et 2). Dans les quartiers où nous avons mené notre enquête, $75 \%$ des ménages ont été victimes des inondations de 2013 à Banconi, contre 65\% à Djicoroni-Para et 30\% à Missabougou.

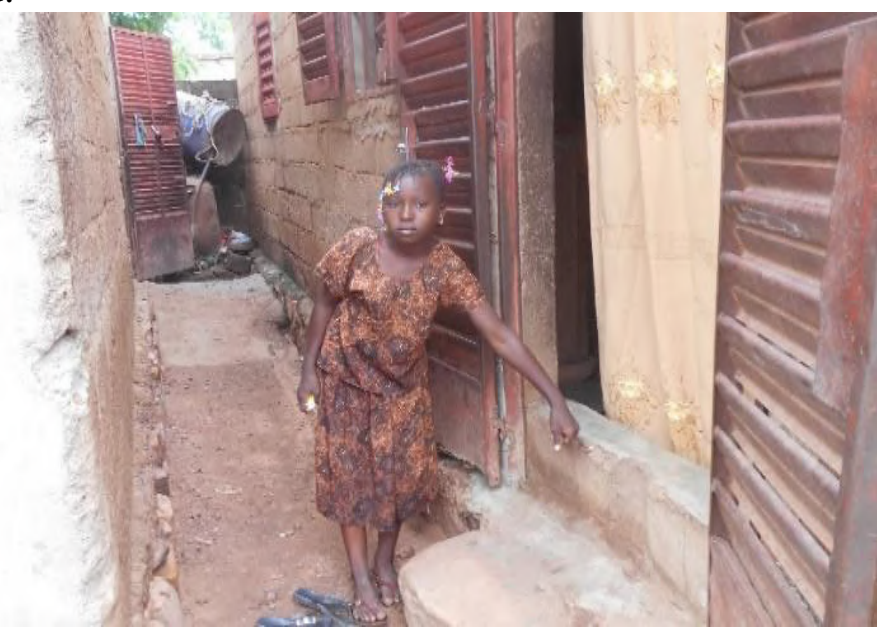

Photo 1 : Une fillette devant une concession dont l'entrée est surélevée pour empêcher la montée des eaux d'entrer dans le bâtiment à Banconi

Source : Cliché, Oumar DEMBELE, Juillet 2016 


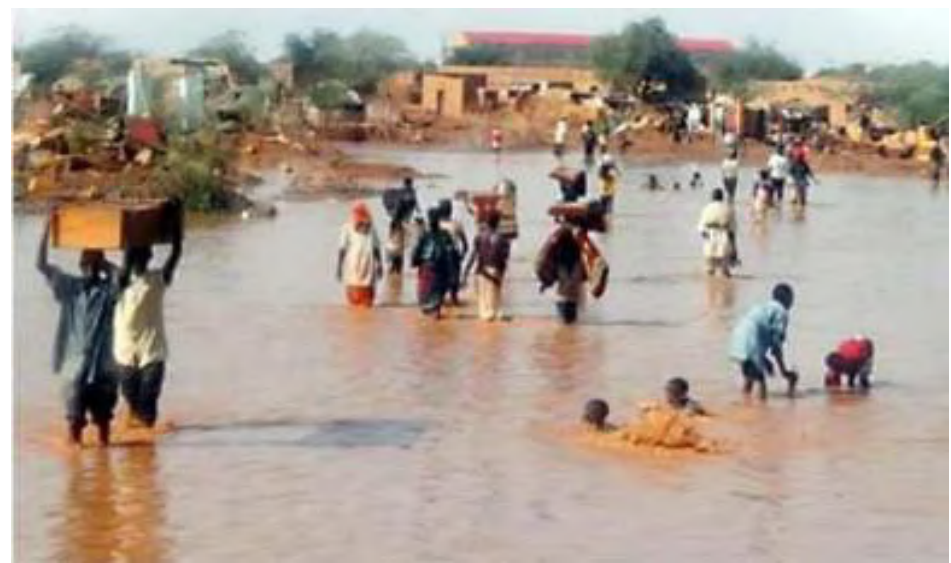

Photo 2 : L’agglomération de Bamako inondée, dont la population sinistrée tente de récupérer les objets emportés par l'eau

Source : Cliché OMS-Mali, 06 septembre 2013

La photo 1 illustre une fillette devant une concession dont l'entrée est surélevée pour empêcher la montée des eaux d'entrer dans le bâtiment dans le quartier populaire de Banconi. Ces inondations qui se sont produites le mercredi 28 août 2013 un peu partout à Bamako, ont été particulièrement meurtrières dans le quartier populaire de Banconi. Elles ont également entrainé d'importants dégâts matériels. Nous avons interviewé Rokia Traoré à Banconi, une victime des inondations. Voici les propos qu'elle nous a tenus : "Nous sommes installés au bord du marigot, nous sommes les premières victimes en cas d'inondation. Des écoulements s'étalent dans les concessions et à l'intérieur des maisons quand il pleut un tout petit. Par exemple, la pluie soudaine survenue en 2013 a provoqué des dommages chez nous ici à Banconi. Les dommages sont énormes, des maisons tombées et des matériels emportés par l'eau et même des pertes de vie humaine ont été enregistrés». A la même année les inondations ont causé à Bamako et particulièrement au quartier Banconi plus de 50 pertes de vie humaines (juillet 2016). La cartes 3, 4 et 5 qui suivent localisent des ménages qui ont été victimes des inondations respectivement dans les quartiers de Djicoroni-Para, Banconi et Missabougou selon notre collecte des données sur le terrain. 


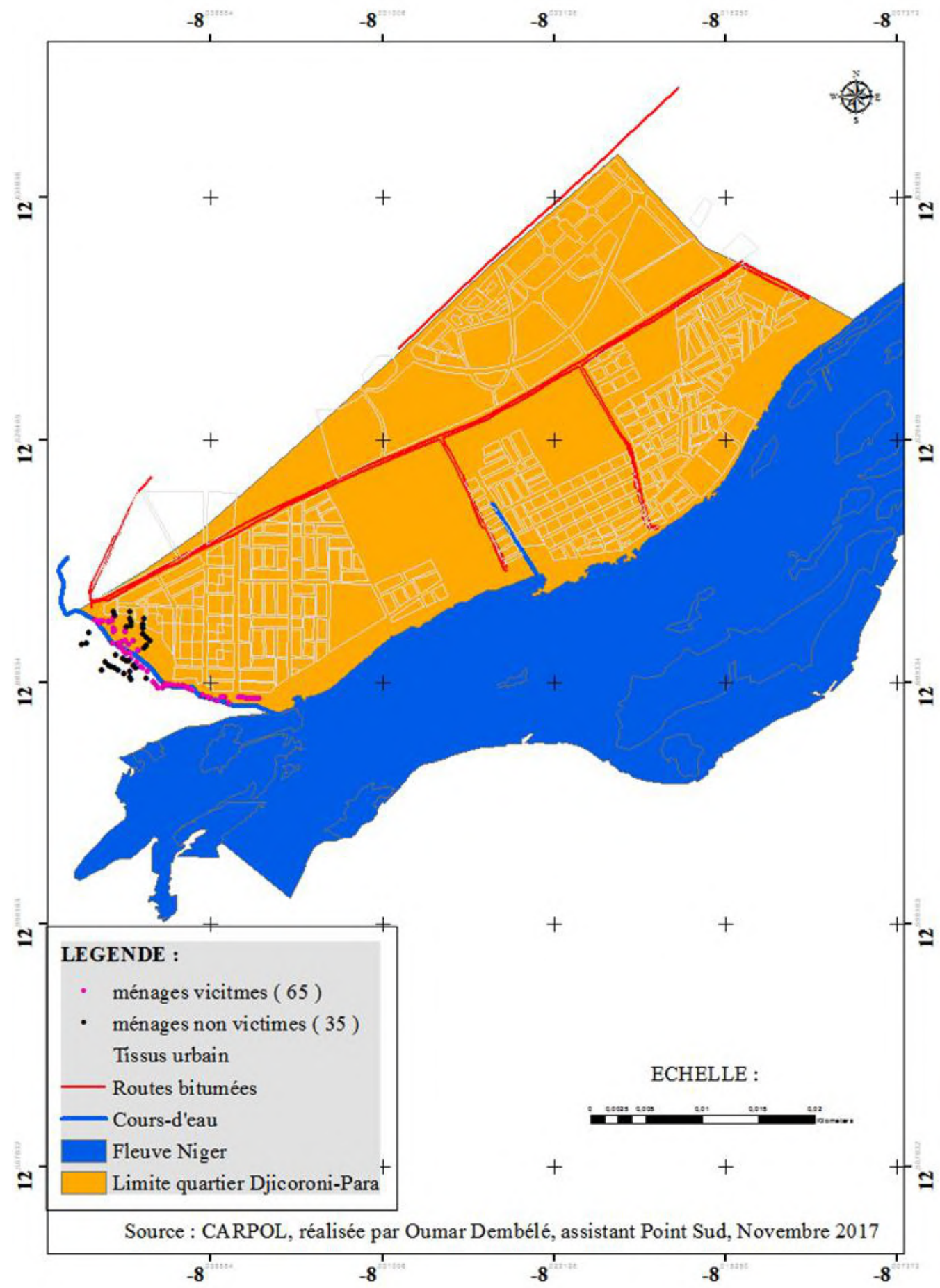

Carte 3 : Localisation des ménages victimes des inondations à Djicoroni-para 


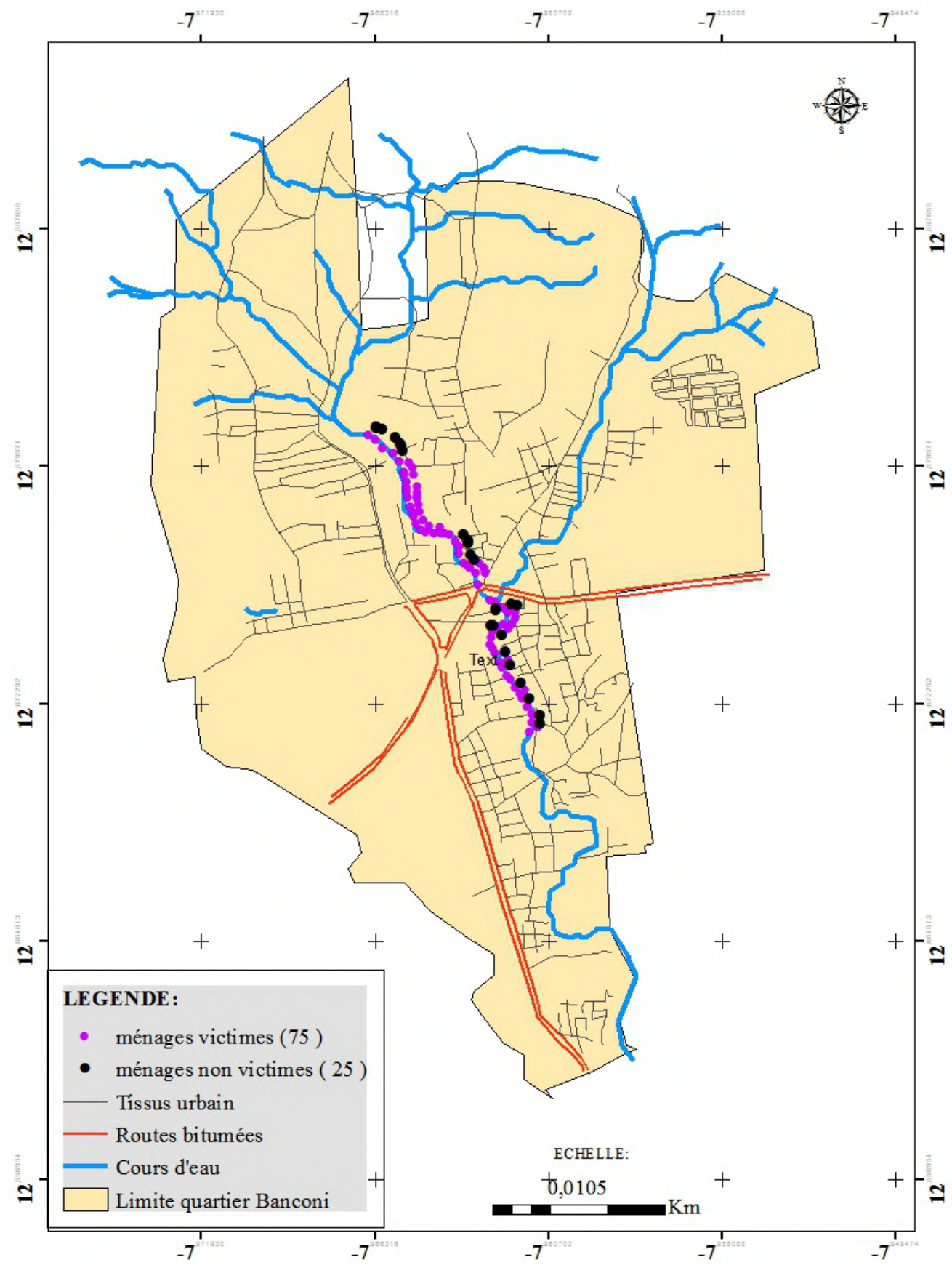

Source : CARPOL, réalisée par Oumar Dembélé, assistant Point Sud , Novembre 2017

Carte 4 : Localisation des ménages victimes des inondations à Banconi 


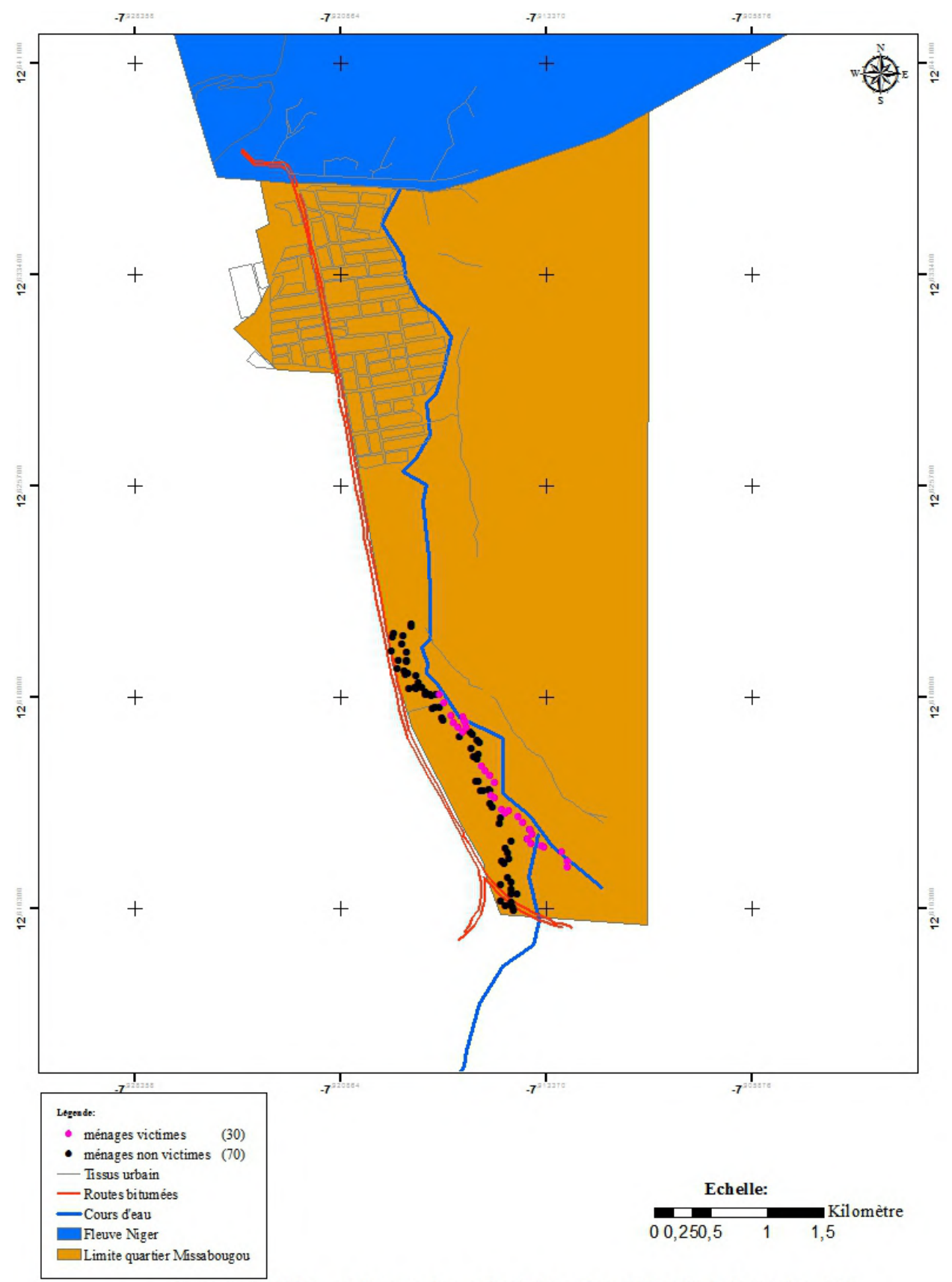

Source: CARPOL, réalisée par Oumar Dembélé, assistant Point-Sud, Novembre 2017

Carte 5 : Localisation des ménages victimes des inondations à Missabougou

\subsubsection{Analyse de la gestion institutionnelle des inondations à Bamako}

En cas de catastrophe majeure, lorsqu'il s'agit de secourir les populations, l'autorité de l'Etat est mieux placée. La Direction Générale de la 
Protection Civile (DGPC), rattachée au Ministère de la Sécurité et de la Protection Civile entre donc en scène et peut déclencher le Plan national d'organisation des secours (plan ORSEC). Au regard des inondations passées à Bamako, les institutions communales, nationales et internationales, ainsi que des agents de santé interviennent «individuellement», principalement pour venir en aide aux populations sinistrées. Ces actions d'urgence s'apparentent à de l'aide humanitaire. Les contraintes majeures portent sur : l'insécurité foncière, le développement anarchique de l'habitat. Dans la gestion des risques d'inondation, une politique de planification urbaine préventive implique donc une réduction de la vulnérabilité par une stratégie d'atténuation des effets des phénomènes naturels et surtout par une amélioration des réponses individuelles et collectives.

\subsubsection{Réponses adaptatives des populations de Bamako : perceptions des inondations, contribution à la vulnérabilité}

La représentation du risque joue sur les stratégies de gestion locales ; et ce, aussi bien individuellement que collectivement (Laganier, 2006). Dans certains quartiers, la saison des pluies signifie automatiquement inondation. L'augmentation des surfaces envahies est constatée, ainsi que l'allongement des périodes. Cette situation peut s'expliquer par l'accroissement des pluies ces dernières années. Les pratiques dans les quartiers sinistrés à Bamako sont évocatrices des problèmes vécus par les populations ainsi que de la manière de les appréhender. Pour tenter de faire face aux inondations et éviter leurs dégâts, les populations mettent en place des réponses spontanées ou relativement adaptatives. Des habitants riverains forment des digues à l'aide de sacs en plastique remplis pour stabiliser l'eau. Cette stratégie n'a jamais porté fruit durant des années car, les cours d'eau sont devenus un dépotoir d'ordures des populations. De ce fait, les tas d'immondices deviennent la trajectoire de l'eau et le lit des cours d'eau s'élargit par conséquent. Certaines pratiques sont néfastes : les populations accentuent leur propre vulnérabilité. Mais à leur décharge, dans le contexte d'une gestion du risque mal maîtrisée, les populations se retrouvent souvent seules. La perception de la gestion des inondations par les autorités participe à leur vulnérabilité. Certains estiment qu'il est du devoir de la municipalité et de l'Etat de réaliser des ouvrages d'assainissement et secourir les sinistrés. Les populations passent alors du désarroi face à l'aléa au sentiment d'abandon et à la colère vis-à-vis des autorités. Elles profitent de la présence de personnes extérieures au quartier pour se faire entendre et accuser les politiques de ne rien faire pour elles. Lors des visites de terrain, par exemple, les populations interpellent et demandent plus généralement de l'aide. Le renforcement des capacités doit alors venir appuyer les populations pour qu'elles soient davantage en mesure de se prémunir contre les impacts occasionnés. Les autorités municipales ont pu 
prendre des dispositions pour le relogement des familles sinistrées. Certaines personnes ont pu être logées dans des écoles. Leur relogement est pris en charge par les autorités et des Organisations Non Gouvernementales (photo 3).

\subsection{Discussion}

Les inondations sont dues à la fois à l'occupation des zones inondables par les populations et par une perturbation des régimes des cours d'eau et des régimes pluviométriques, provoqués par la variabilité du climat. L'agressivité du climat du fait des changements climatiques entraîne une diminution du nombre de jours de pluie ces dernières années, marquée par un retour des précipitations depuis 1994. Les pays, surtout ceux en développement, ne disposent pas de moyens suffisants pour faire face au risque d'inondation. Les résultats obtenus dans le cadre de notre étude ont permis de comprendre que les populations riveraines ne disposent pas de moyens adéquats pour faire face aux situations d'inondation. Par conséquent, la prévention de ce type de risque naturel contribuerait à réduire considérablement les dégâts en cas d’occurrence (Kouamé, 1999, p.24 ; Saley, 2003, p.32).

Notre étude revêt donc tout son intérêt car, elle a permis de spatialiser les habitations installées dans les zones à risque d'inondation, évaluer les conséquences dommageables des populations vulnérables et nécessitant une intervention prioritaire. En effet, une étude similaire a été réalisée par Saley (2003) dans la région semi-montagneuse à l'Ouest de la République de Côted'Ivoire. Les auteurs ont identifié cinq niveaux de risque (très faible, faible, modérée, forte et très forte). Cela s'explique par le fait que Saley (2003) ont travaillé dans une zone très contrastée (altitude comprise entre 200 et $600 \mathrm{~m}$ ). A la différence des résultats des travaux de Saley (2003), nos travaux ont l'avantage d'inclure en plus du risque d'inondation, la vulnérabilité des populations liée à la localisation très rapprochée des habitations aux marigots et à la qualité des bâtis.

\section{Conclusion}

La dynamique spatiale de Bamako, à laquelle la première partie de l'article s'est intéressée, montre une ville changeante, sinon même trop changeante. Ce changement se traduit essentiellement par un étalement horizontal à l'exagération duquel contribuent plusieurs facteurs (problème de logement, spéculation foncière, etc.) y compris culturel comme l'habitude de la maison individuelle et d'une grande cour. Le facteur principal reste cependant, l'accroissement rapide de la population. De façon générale, les résultats permettent de juger la contribution du Système d'Information Géographique (SIG) comme outil d'aide à la prise de décisions au renforcement de la planification préventive des risques naturels et des 
stratégies d'adaptation. Les analyses présentées dans l'étude ont ainsi contribué à mettre en évidence que la dynamique urbaine de Bamako ne s'est pas menée au hasard; elle s'est effectuée à travers des politiques publiques urbaines.

A ce titre, notre étude ressort sur ce postulat le bilan de la mise en œuvre des politiques publiques de planification et de gestion urbaine à nos jours. Les Systèmes d'information géographique grâce à leur capacité, à partir de diverses sources, de rassembler, d'organiser, de gérer, d'analyser, de combiner, d'élaborer, de présenter des informations localisées, ont permis de produire des cartes qui ont servi à l'analyse spatiale de la ville de Bamako. Le recours aux SIG nous a permis encore de constituer une base de données et d'obtenir des informations géolocalisées ou géocodées sur les lits des marigots occupés par les constructions dans la ville de Bamako. Les résultats obtenus permettent de connaitre les zones à risque d'inondation, les populations touchées, leur niveau de vulnérabilité, les moyens utilisés pour faire face et les stratégies d'adaptation.

\section{References:}

1. Bulle, S. (1997). Légitimé et stratégie des acteurs du secteur de l'eau se réclamant d'une représentativité collective : Conditions d'émergence des nouveaux acteurs et diffusion de dynamiques locales, Sénégal, Mali, Haïti, Cameroun, Groupe de Recherche et d'Echange Technologique (GRET), Paris, 126 p.

2. Dia, A.M., Kouamé J., Wade, S., Rudant, J.P. (2006a). Application of Remote Sensing and GIS in natural disaster risk management: Case study of the Senegal river flood monitoring project (GESCAN), Communication and poster to the International Disaster Reduction Conference (IRDC). Davos, Switzerland, August 27th-September 2, 2006.

3. Dia, A.M., Wade, S., Deroin, J.P., Frison, P.L., Kouamé, J., Rudant, J.P. (2006b). Apport des données SPOT et Landsat au suivi des inondations dans l'estuaire du fleuve Sénégal, Photo-interprétation, 2006/4, pp.35-46.

4. Diarra, B. (1999). Dynamique spatiale et politiques urbaines à Bamako : le rôle des images-satellite SPOT dans la gestion des villes, thèse de doctorat, Université Marseille I - Université Ex en Provence, Marseille, $273 \mathrm{p}$.

5. Direction Régionale de la Planification, de la Statistique, de l'Informatique, de l'Aménagement du Territoire et de la Population du District de Bamako. (2015). Annuaire statistique du district de Bamako, année 2014, Bamako, 225 p. 
6. Gosselin, P. (1953). Bamako, ville soudanaise, l'Afrique et l'Asie, $\mathrm{n}^{\circ} 21,1^{\mathrm{er}}$ semestre, pp.31-37

7. Hubert, G., Ledoux, B. (1999). Le coût du risque... L'évaluation des impacts socio-économiques des inondations, Presses de l'Ecole Nationale des Ponts et Chaussées, Paris, 232p.

8. Institut National de la Statistique du Mali. (2009). $4^{\text {ème }}$ Recensement Général de la Population et de l'Habitat du Mali-RGPH, analyse des résultats définitifs : état et structure de la population, rapport final, 87 p.

9. Kouamé, K.F. (1999). Hydrogéologie des régions de montagne : apports des données de télédétection et des méthodes statistique et fractale à l'élaboration d'un Système d'Information Hydrogéologique à Référence Spatiale des aquifères discontinus du secteur Man-Danané (Ouest de la Côte d'Ivoire), thèse de doctorat, Université de Cocody, Bamako, 210 p.

10. Laganier, R. (2006). Territoires, inondation et figures du risque : la prévention au prisme de l'évaluation, l'Harmattan, coll. 'Itinéraires géographiques", Paris, $257 \mathrm{p}$.

11. Ministère de l'Urbanisme et de l'Habitat du Mali. (1995). Schéma Directeur d'Aménagement et d'Urbanisme de Bamako et environs, Bamako, $2^{\text {ème }}$ révision, $112 \mathrm{p}$.

12. Ozer, P. (2014). Catastrophes naturelles et aménagement du territoire : de l'intérêt des images Google Earth dans les pays en développement, Géo-Eco-Trop, n³8, pp.209-220.

13. Pottier, N. (2003). La lutte contre les inondations en France : outils et stratégies d'hier à demain. Les risques, éditions du Temps, collection Géographie, pp.173-204.

14. Saley, M.B. (2003). Système d'Information Hydrogéologique à Référence Spatiale, discontinuités, pseudo images et cartographie thématique des ressources en eau de la région montagneuse de Man (Ouest de Côte-d'Ivoire), thèse de doctorat, Université de Cocody, Abidjan, $211 \mathrm{p}$.

15. Thénot, A. (2007). Modèles de données pour l'appréhension et la gestion des risques à Nouakchott (Mauritanie) - Une capitale contre vents et marées, thèse de doctorat, Université Panthéon-Sorbonne, Paris I, 424 p.

16. Traoré, D., Sy, I., Utzinger, J., Epprecht, M., Kengne, I.M., Lô, B., Odermatt, P., Faye, O., Cissé, G., Tanner, M. (2013). Water quality and health in a Sahelian semi-arid urban context: an integrated geographical approach in Nouakchott, Mauritania, Geospatial Health, $\mathrm{n}^{\circ} 8$, pp.53-63. 
17. Villien-Rossi, M.L. (1963). Bamako, capitale du Mali, Cahier d'OutreMer, n64, pp.379 - 393.

18. Villien-Rossi, M.L. (1966). Les kindas de Bamako, Cahier d'OutreMer, $\mathrm{n}^{\circ} 76, \mathrm{pp} .364-381$.

19. Villien-Rossi, M.L. (1966). Bamako, capitale du Mali, Bulletin IFAN, tome $28, \mathrm{n}^{\circ} 1 / 2, \mathrm{pp} .248-378$. 